\title{
Correction to: MiRNA-126 expression inhibits IL-23R mediated TNF-a or IFN- $\gamma$ production in fibroblast-like synoviocytes in a mice model of collagen-induced rheumatoid arthritis
}

\author{
Jie Gao ${ }^{1} \cdot$ Ruina Kong $^{1} \cdot$ Xiaoli Zhou $^{2} \cdot$ Lianmei Ji $^{1} \cdot$ Ju Zhang $^{1} \cdot$ Dongbao Zhao $^{1}$
}

Published online: 12 January 2019

(c) The Author(s) 2019

\section{Correction to: Apoptosis (2018) 23:607-615 \\ https://doi.org/10.1007/s10495-018-1474-7}

The authors would like to add an article note stating that

"The authors Jie Gao and Ruina Kong have equally contributed to the article".

Jie Gao and Ruina Kong have contributed equally to the article.

The original article can be found online at https://doi.org/10.1007/ s10495-018-1474-7.

Dongbao Zhao

dongbaozhao@163.com

1 Department of Rheumatology and Immunology, Changhai Hospital, Second Military Medical University, No. 168 Changhai Road, Shanghai 200433, China

2 Department of Pathology, Changzhou Second People's Hospital, Changzhou, China 